\title{
Perioperative care of the older patient
}

E. Blommers ${ }^{1}$, M. Klimek ${ }^{1}$, K.A. Hartholt ${ }^{2,3}$, T.J.M. van der Cammen ${ }^{2}$, J. Klein ${ }^{1}$, P.G. Noordzij ${ }^{1}$

${ }^{1}$ Department of Anesthesiology

${ }^{2}$ Section of Geriatric Medicine-Department of Internal Medicine

${ }^{3}$ Department of Surgery-Traumatology

Erasmus MC, University Medical Center Rotterdam, Rotterdam, The Netherlands

Corresponding author

P.G. Noordzij, MD, PhD

Erasmus MC, University Medical Center Rotterdam

Department of Anesthesiology

Room H12-N

PO Box 2040

3000 CA Rotterdam, The Netherlands

E-mail: peternoordzij@me.com

Date: August 16, 2011 
Conflict of Interest

No conflict of interest has been reported for any of the authors. 


\section{Abstract}

Nearly $60 \%$ of the Dutch population undergoing surgery is aged 65 years and over. Older patients are at increased risk of developing perioperative complications (e.g. myocardial infarction, pneumonia or delirium), which may lead to a prolonged hospital stay or death. Preoperative risk stratification calculates a patient's risk by evaluating the presence and extent of frailty, pathophysiological risk factors, type of surgery and the results of (additional) testing. Type of anesthesia, fluid management and pain management affect outcome of surgery. Recent developments focus on multimodal perioperative care of the older patient, using minimal invasive surgery, postoperative anesthesiology rounds and early geriatric consultation.

Keywords Perioperative care, older adults, complications, PCOD 
Introduction

Countries worldwide are facing the challenge of an aging population. Because of improved medical care, the life expectancy increased over the last decades and is expected to increase further. The population of persons aged $\geq 65$ years will double in the coming three to four decades. According to Dutch national healthcare statistics, $25 \%$ of the estimated 17 million inhabitants will then be aged 65 years and over. Approximately $4 \%$ of the Dutch population is scheduled for a surgical procedure, which requires hospitalization, annually. Currently, almost $60 \%$ of these patients are aged $\geq 65$ years.

Older patients are at increased risk of complications during the perioperative period for several reasons. Firstly, physiological aging results in a diminished ability of the body to cope with stress associated with surgery and hospitalization. These physiological changes affect pharmacokinetics and pharmaco-dynamics of many drugs. Secondly, advanced age is accompanied by an increased prevalence of comorbidities and subsequent increase of perioperative risk. Most morbidity and mortality in the perioperative setting is caused by the vital body functions in the cardiovascular and pulmonary system. In this paper we will highlight the main perioperative risk factors in older adults.

\section{Preoperative risk factors}

\section{Cardiovascular complications}

Ageing of the cardiovascular system results in a decreased compliance of the heart and blood vessels and a diminished response to stimulation of beta adrenergic receptors in the myocardium. ${ }^{1}$ Older adults are susceptible to diastolic heart failure and ventricular function relies on adequate atrial contraction and a normal blood volume. As a result, intraoperative changes in circulating blood volume are associated with an increased risk of hemodynamic instability. 
One third of perioperative morbidity and mortality is caused by cardiovascular complications (e.g. myocardial infarction, arrhythmias, heart failure and stroke). ${ }^{2}$ Perioperative cardiovascular risk stratification is based on clinical risk factors and type of surgical procedure (Table 1 and 2). In patients at increased risk, the presence and extent of coronary disease can be evaluated by additional pre-operative testing (Figure 1).

In a randomized controlled trial of 510 patients undergoing elective vascular surgery, preoperative coronary revascularization was not associated with a reduction in postoperative myocardial ischemia (12\% versus $14 \%$ in patients without preoperative revascularization; $p=0.37)$ or death $(22 \%$ versus $23 \% ; p=0.92) .{ }^{3}$ Current guidelines indicate a class $\mathrm{lb}$ recommendation of perioperative beta-blockade in patients scheduled for vascular surgery, with myocardial ischemia on preoperative testing, and a lla recommendation for intermediate risk patients $(<1$ clinical risk factor) undergoing intermediate risk or vascular surgery. ${ }^{4}$ However, in a recent multicenter trial of 8,351 intermediate risk patients undergoing major non-cardiac surgery, perioperative high-dose metoprolol succinate treatment was associated with a $33 \%$ increased risk of overall mortality and a $117 \%$ increased risk of stroke, despite a $30 \%$ reduction in the risk of nonfatal myocardial infarction. ${ }^{5}$ These data demonstrate the need for further studies.

\section{Pulmonary complications}

Increased age is associated with a reduced respiratory drive and a decreased compliance of thorax and lungs. ${ }^{6}$ A reduction in cardiac output and in functionality of the lung parenchyma results in an increased alveolar-arterial oxygen gradient and a limited respiratory compensation capacity. As a result, older patients are at risk for hypoxia and exhaustion at times of increased work of breathing.

Frequent postoperative pulmonary complications, e.g. pneumonia, exacerbation of asthma or chronic obstructive pulmonary disease (COPD) and 
respiratory failure, are associated with an increased length of hospital stay and mortality. Important risk factors are: increased age ( $\geq 60$ years), COPD, current smoking, heart failure, decreased exercise tolerance, higher ASA score (American Society of Anesthesiologists physical status classification for assessing a patient prior to surgery), abdominal-thoracic surgery, increased duration of procedure ( $>3$ hours), general anesthesia and urgent surgery. ${ }^{7}$ The additional value of preoperative spirometry, chest radiography and blood gas analysis is controversial and should not be used routinely. Table 3 shows several intervention strategies to reduce postoperative pulmonary complications.

\section{Metabolic complications}

Ageing is associated with a reduction of body water volume, renal mass, renal perfusion and glomerular filtration rate and a relative increase of body fat mass. As a result, renal function is reduced, which is associated with increased risk of volume overload, electrolyte disorders and accumulation of certain drugs (e.g. morphine, pancuronium, rocuronium and digoxin). NSAIDs, diuretics and antibiotics are associated with an increased risk of acute kidney injury, especially in hypovolemic patients.

Malnutrition is a common problem in the older population. The presence and extent of postoperative catabolism is associated with an increased length of hospital stay, pulmonary complications and risk of infectious disease ${ }^{7,8}$ A systematic review on postoperative care in older patients after hip fracture surgery, showed a reduction in postoperative complications with additional oral feeding of the patient (relative risk $0.52 ; 95 \%$ confidence interval $0.32-0.84) .{ }^{9}$ However, further research is warranted.

\section{Anesthesiological aspects}

Preoperative patient information 
Informing patients about a planned surgical procedure and associated hospitalization influences their perception of the event. Preoperative instruction has been demonstrated to reduce patient anxiety, postoperative pain and length of hospital stay. ${ }^{10}$ However, preoperative teaching (e.g. instructional videos, information meetings, preoperative visit, World Wide Web) should be tailored according to a patient's coping behaviour and intellectual capacity as they can vary considerably.

\section{Premedication}

Preoperative sedatives or analgesics seem to have little effect on postoperative anxiety levels of the patient, length of hospital stay or pain perception. ${ }^{11,12}$ However, short acting intravenous anesthetics, administered by an anaesthesiologist at the operating theatre, can be of additional value to reduce anxiety in patients while performing central or regional nerve blocks.

\section{Neuraxis blockade}

Irrespective of type of surgery, postoperative epidural analgesia provides superior pain relieve compared to intravenous administered opioids up to 4 days after surgery. ${ }^{13}$ In patients undergoing major vascular surgery, thoracic epidural analgesia has been associated with a reduced risk of myocardial infarction $(2.7 \%$ versus $7.9 \%$, $\mathrm{p}=0.05$ ) and postoperative pulmonary complications (14 versus $28 \%, \mathrm{P}<0.01) .{ }^{14} \mathrm{~A}$ meta-analysis of 9,559 patients, undergoing different types of surgery, showed a reduced postoperative mortality rate associated with epidural analgesia compared with general anesthesia alone ( 1.9 versus $2.8 \%$; odds ratio 0.7 and $95 \%$ confidence interval 0.54-0.9). ${ }^{15}$ However, these favourable result could not be confirmed by a systematic review and a randomized trial. ${ }^{16,17}$ 


\section{Fluid management}

Perioperative hypovolemia, due to e.g. pre-existing malnutrition, preoperative fasting, bowel preparation or intraoperative blood loss, is a common problem and may be an important determinant of an increased length of hospital stay, morbidity and mortality. Several randomized trials have associated preoperative intravascular volume optimization, guided by Doppler monitoring or pulse pressure variation, with a reduction of length of hospital stay varying from 2 to 10 days, mainly due to shorter duration of mechanical ventilation and intensive care unit stay, earlier return of bowel function and lower incidence of postoperative nausea and vomiting. These beneficial effects were more pronounced in older patients and patients with comorbidities. ${ }^{18-20}$ In a review of 9 trials studying 10 types of fluids (colloids and crystalloids) in 412 patients undergoing aortic surgery, no single fluid was shown to affect outcome measures of the patient. ${ }^{21}$

\section{Postoperative pain management}

Despite and/or due to an increased frequency and more intense perception of postoperative pain, undertreatment of pain is common in older patients. Optimized pain relief allows early mobilization and is a prerequisite for fast recovery. ${ }^{22}$ Pain is not only uncomfortable, but also brings with it increased risk in the cardiovascular system, such as a stress tachycardia and even delirium. Postoperative pain management depends on type of surgery and a patient's comorbidities. ${ }^{22}$ In case of low and intermediate postoperative pain, paracetamol is an effective basic analgesic, which has few contraindications. Postoperative NSAIDs reduce the need of opioid treatment. However, caution is advised in patients with hypertension, renal insufficiency and heart failure if NSAIDs are used over a longer period. Cyclooxygenase (COX)-2-selective NSAIDs affect renal function similarly to non-selective NSAIDs and have recently been associated with an increased risk of thromboembolic complications in patients with cardiovascular disease. ${ }^{23}$ 
Opioids are potent analgesics, which are used in case of severe postoperative pain. The old are especially susceptible to side effects of opioid treatment, e.g. constipation, decreased respiratory drive and sedation, which could lead to choking and pneumonia. Opioid treatment in older adults is associated with a more potent and longer lasting effect, which is even more pronounced in case of lower serum albumin levels. Patient controlled analgesia results in rapid, predictable pain treatment and superior patient satisfaction, compared to intramuscular opioid injections. ${ }^{24}$ Intramuscular injections should not be done in critically ill patients. In general, intramuscular injections of opioids should be avoided, because subcutaneous injections show the same efficacy, with a minor risk of side effects.

\section{Postoperative cognitive dysfunction}

Acute postoperative delirium (POD) is characterized by sudden disorientation associated with changes in cognitive behavior and consciousness, with disturbed psychomotor activity.POD is a temporary and reversible cognitive disturbance occurring immediately after surgery and short-time existing. The majority of acute deliria occur between the first and seventh postoperative day and are associated with an increased length of hospital stay, prolonged recovery and higher postoperative morbidity and mortality rates. ${ }^{25}$ The prevalence of acute POD averages $10 \%$ of the surgical population, but increases to $30-60 \%$ in patients undergoing high risk surgery or urgent procedures. Table 4 shows several risk factors associated with acute postoperative delirium.

Post operative Cognitive Dysfunction (POCD) is a temporary and delayed reversible cognitive disturbance with a duration more than a week. POCD is associated with a cognitive decline during months after surgery. However, the clinical symptoms are not as severe as in POD. Etiology, risk factors and consequences of long-term POCD are unknown in patients undergoing non-cardiac surgery. In a prospective multicenter trial of 1,218 patients undergoing major abdominal, non- 
cardiac thoracic or orthopedic surgery, the occurrence of long-term POCD and associated risk factors in older patients was investigated. POCD was present in 9,9\% of patients at 3 months after surgery, compared to $2,8 \%$ in controls. Age was the only risk factor associated with long-term POCD. ${ }^{26}$

The effect of type of anesthesia on the incidence of POCD is limited. In a study of 262 older patients scheduled for elective primary total knee replacement, patients were randomly assigned to either epidural or general anesthesia. Overall, $5 \%$ showed clinically significant long-term POCD. Type of anesthesia did not influence the incidence or magnitude of POCD. ${ }^{27}$ A structured protocol reduced delirium incidence with one third in patients after hip fracture surgery, due to proactive geriatric consultation and treatment of frequent postoperative problems, e.g. pain, anemia, hypoxia, electrolyte disturbances, dehydration, malnutrition, immobilization and early treatment of signs of delirium. ${ }^{28}$ In a randomized trial on the effect of low-dose haloperidol prophylaxis on the incidence of delirium in orthopedic patients, $1.5 \mathrm{mg}$ haloperidol daily until 3 days after surgery, was not associated with a significant reduction in the incidence of postoperative delirium compared to placebo (15.1\% versus $16.5 \%$ respectively). However, patients treated with haloperidol prophylaxis showed a reduction of severity and duration of delirium. Importantly, length of hospital stay in patients on haloperidol prophylaxis was reduced with 5.5 days $(p<0.001){ }^{29}$

\section{Proactive geriatric consultation}

Improved perioperative recovery includes many components, and a multidisciplinary approach between anesthesiologists, surgeons, pharmacists, geriatricians and physiotherapists is needed. Specialized 'in-hospital tracks' have been developed for patient with hip fractures with promising results and leading to an accelerated recovery. Pre- and postoperative anesthetic rounds with an anesthesiologist and a geriatrician can unmask potential complications and anticipate on the risk factors. 
Multidisciplinary teams, with early geriatric consultation are leading to reduced admission duration and fewer complications in older patients. ${ }^{30-32}$ Thus, although a single individual or department can initiate such an approach, it will only succeed with active and committed participation of other healthcare professionals.

\section{Concluding remarks}

Care of the older surgical patient is an important part of perioperative medicine. In this respect, knowledge of specific pathophysiological and pharmacological aspects of this group of patients is needed in order to guarantee quality assurance. A combination of surgical, geriatric and anesthesiological factors seems to be responsible for a prolonged hospital stay and an increased morbidity and mortality. Recent developments focus on early recovery by multimodal perioperative care ${ }^{30-32}$ which includes minimal invasive surgery, postoperative anesthesiology rounds, and proactive geriatrical consultation. Anesthetists and geriatricians should be aware of the responsibility and opportunities for the development and successful implementation of such programs in daily perioperative practice. 


\section{Acknowledgments}

Klaas Hartholt is a research fellow at the Erasmus MC, appointed on a research grant from "The Netherlands Organization for Health Research and Development" (ZonMw), project number 170.885.607. 
Table 1. Clinical predictors of increased perioperative cardiovascular risk ${ }^{*}$

\section{Major}

Unstable coronary syndromes

Acute or recent myocardial infarction with evidence of important ischemic risk by

clinical symptoms or non-invasive study**

Unstable or severe angina (Canadian class III or IV)

Decompensated heart failure

Significant arrhythmias

High-grade atrioventricular block

Symptomatic ventricular arrhythmias in the presence of underlying heart disease

Supra ventricular with uncontrolled ventricular rate

Severe valvular disease

\section{Intermediate}

Mild angina pectoris (Canadian Class I or II)

Previous myocardial infarction by history of pathological $Q$ waves

Compensated or prior heart failure

Diabetes mellitus (particularly insulin dependent)

Renal insufficiency

\section{Low}

Advanced age

Abnormal electrocardiogram (left ventricular hypertrophy, left bundle-branch block,

ST-T abnormalities)

Rhythm other than sinus (e.g. atrial fibrillation)

Low functional capacity

History of stroke

Uncontrolled systemic hypertension

${ }^{*}$ Combined incidences of cardiac death, nonfatal myocardial infarction

${ }^{* *}$ Recent myocardial infarction defined as $>7$ days, $\leq 1$ month 
Tabel 2. Cardiac risk stratification for noncardiac procedures*

\section{High}

Emergent major operations, particularly in the older

Aortic and other major vascular surgery

Peripheral vascular surgery

Anticipated prolonged surgical procedures associated with large fluid shifts

\section{Intermediate}

Carotid endarterectomy

Head and neck surgery

Intraperitoneal and intrathoracic surgery

Orthopedic surgery

Prostate surgery

\section{Low}

Endoscopic procedures

Superficial procedures

Cataract surgery

Breast Surgery

${ }^{*}$ Combined incidence of cardiac death and nonfatal myocardial infarction High: reported cardiac risk $>5 \%$,

Intermediate: reported cardiac risk $<5 \%$,

Low: reported cardiac risk $<1 \%$ 
Table 3. Strategies for perioperative pulmonary risk reduction

\section{Preoperative}

Corticosteroid treatment in patients with reversible airway obstruction*

Cessation of smoking 6 - 8 weeks prior to surgery**

\section{Intraoperative}

Neuraxis blockade compared to general anesthesia*

Short- or intermediate acting muscle relaxants*

Selective use of nasogastric tube after elective laparotomy*

Minimal invasive surgery ${ }^{\star *}$

Early extubation**

\section{Postoperative}

Lung recruitment therapy (e.g. incentive spirometry, deep breathing exercises) ${ }^{*}$

Epidural analgesia*

Corticosteroid treatment in patients with reversible airway obstruction*

${ }^{*}$ Associated with a reduction in pulmonary complications

${ }^{\star *}$ Probably associated with a reduction in pulmonary complications, further evidence needed 
Table 4. Risk factors associated with postoperative delirium

\section{Patient related risk factors}

Age $\geq 70$

Preexisting mild cognitive dysfunction

Polypharmacy

Use of psychopharmaca

Higher ASA score

Dementia

Depression

Alcohol abuse

Malnutrition

Procedure related risk factors

Type of surgery

Increased duration of surgery

Re-operation

Large intraoperative blood loss

Postoperative anemia

Polytransfusion

Use of anticholinergics

\section{Other risk factors}

Medical complications

Sepsis

ICU admission 
Figure 1. Cardiac risk stratification and optimisation of surgical patients

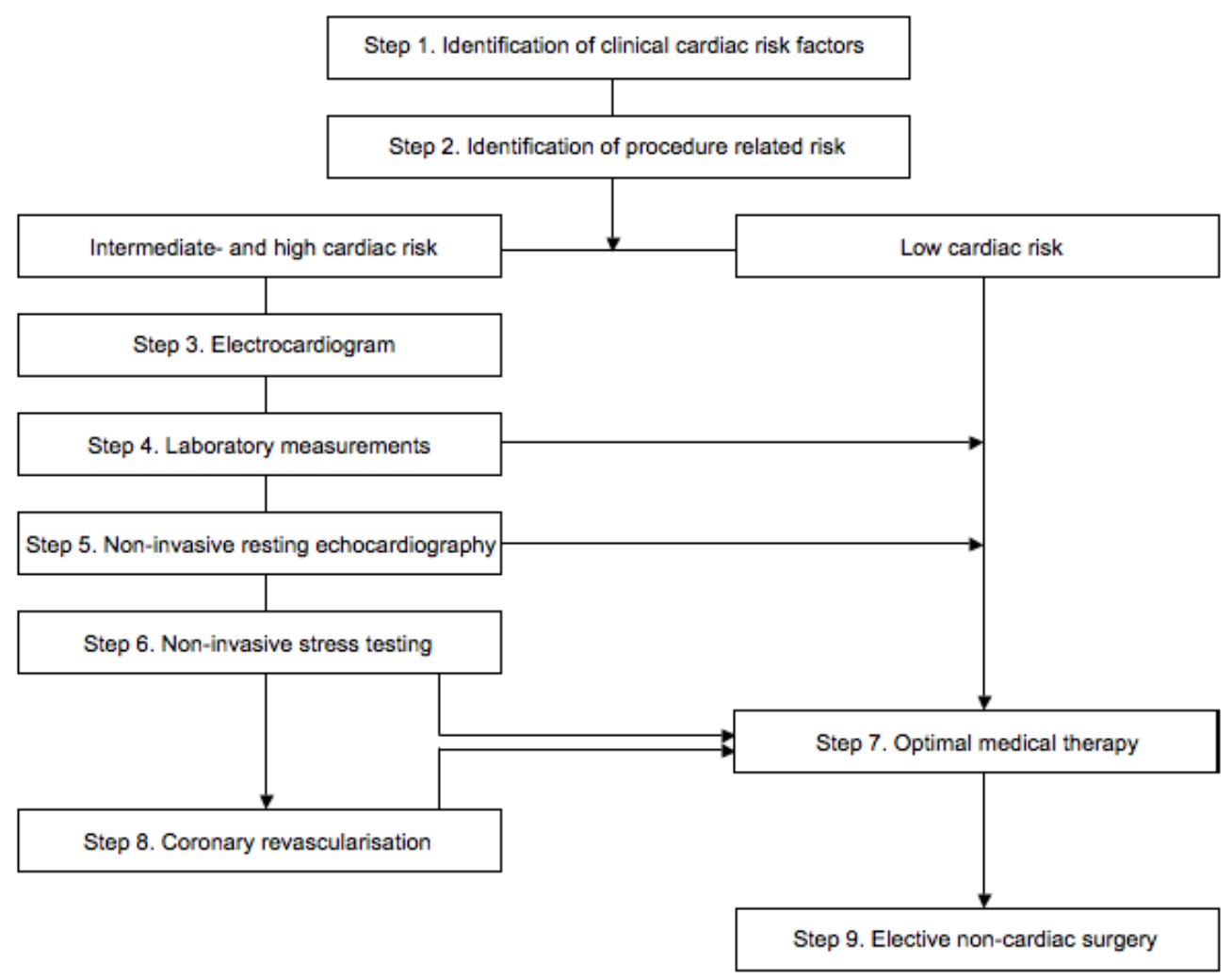




\section{References}

1. Rooke GA. Cardiovascular aging and anesthetic implications. J Cardiothorac Vasc Anesth 2003;17(4):512-23.

2. Lee TH, Marcantonio ER, Mangione CM, et al. Derivation and prospective validation of a simple index for prediction of cardiac risk of major noncardiac surgery. Circulation 1999;100(10):1043-9.

3. McFalls EO, Ward HB, Moritz TE, et al. Coronary-artery revascularization before elective major vascular surgery. N Engl J Med 2004;351(27):2795-804.

4. Fleisher LA, Beckman JA, Brown KA, et al. ACC/AHA 2007 Guidelines on Perioperative Cardiovascular Evaluation and Care for Noncardiac Surgery: Executive Summary. J Am Coll Cardiol 2007;50(17):1707-32.

5. Devereaux PJ HO. The perioperative ischemic evaluation (POISE) trial: a randomized controlled trial of metoprolol versus placebo in patients undergoing noncardiac surgery. Clin Res Cardiol 2008;97(8).

6. Allen S. Respiratory considerations in the elderly surgical patiënt. Clinics in Anesthesiology 1986;4:899-930.

7. Qaseem A, Snow V, Fitterman N, et al. Risk assessment for and strategies to reduce perioperative pulmonary complications for patients undergoing noncardiothoracic surgery: a guideline from the American College of Physicians. Ann Intern Med 2006;144(8):575-80.

8. Thorell A, Nygren J, Ljungqvist O. Insulin resistance: a marker of surgical stress. Curr Opin Clin Nutr Metab Care 1999;2(1):69-78.

9. Avenell $\mathrm{A}$, Handoll $\mathrm{HH}$. Nutritional supplementation for hip fracture aftercare in older people. Cochrane Database Syst Rev 2006(4):CD001880.

10. Klafta JM, Roizen MF. Current understanding of patients' attitudes toward and preparation for anesthesia: a review. Anesth Analg 1996;83(6):1314-21.

11. Caumo W, Schmidt AP, Schneider CN, et al. Risk factors for postoperative anxiety in adults. Anaesthesia 2001;56(8):720-8.

12. Moiniche $\mathrm{S}$, Kehlet $\mathrm{H}$, Dahl JB. A qualitative and quantitative systematic review of preemptive analgesia for postoperative pain relief: the role of timing of analgesia.

Anesthesiology 2002;96(3):725-41.

13. Block BM, Liu SS, Rowlingson AJ, Cowan AR, Cowan JA, Jr., Wu CL. Efficacy of postoperative epidural analgesia: a meta-analysis. Jama 2003;290(18):2455-63.

14. Park WY, Thompson JS, Lee KK. Effect of epidural anesthesia and analgesia on perioperative outcome: a randomized, controlled Veterans Affairs cooperative study. Ann Surg 2001;234(4):560-9; discussion 9-71.

15. Rodgers A, Walker N, Schug S, et al. Reduction of postoperative mortality and morbidity with epidural or spinal anaesthesia: results from overview of randomised trials. Bmj 2000;321(7275):1493.

16. Liu SS, Wu CL. Effect of postoperative analgesia on major postoperative complications: a systematic update of the evidence. Anesth Analg 2007;104(3):689-702. 17. Peyton PJ, Myles PS, Silbert BS, Rigg JA, Jamrozik K, Parsons R. Perioperative epidural analgesia and outcome after major abdominal surgery in high-risk patients. Anesth Analg 2003;96(2):548-, table of contents.

18. Lopes MR, Oliveira MA, Pereira VO, Lemos IP, Auler JO, Jr., Michard F. Goaldirected fluid management based on pulse pressure variation monitoring during high-risk surgery: a pilot randomized controlled trial. Crit Care 2007;11(5):R100.

19. Sinclair S, James S, Singer M. Intraoperative intravascular volume optimisation and length of hospital stay after repair of proximal femoral fracture: randomised controlled trial. Bmj 1997;315(7113):909-12.

20. Gan TJ, Soppitt A, Maroof M, et al. Goal-directed intraoperative fluid administration reduces length of hospital stay after major surgery. Anesthesiology 2002;97(4):820-6.

21. Whatling PJ. Intravenous fluids for abdominal aortic surgery. Cochrane Database Syst Rev 2000(4):CD000991. 
22. Kehlet H, Dahl JB. Anaesthesia, surgery, and challenges in postoperative recovery. Lancet 2003;362(9399):1921-8.

23. Solomon SD, McMurray JJ, Pfeffer MA, et al. Cardiovascular risk associated with celecoxib in a clinical trial for colorectal adenoma prevention. $\mathrm{N}$ Engl J Med 2005;352(11):1071-80.

24. Egbert AM, Parks LH, Short LM, Burnett ML. Randomized trial of postoperative patient-controlled analgesia vs intramuscular narcotics in frail elderly men. Arch Intern Med 1990;150(9):1897-903.

25. Marcantonio ER, Goldman L, Mangione CM, et al. A clinical prediction rule for delirium after elective noncardiac surgery. Jama 1994;271(2):134-9.

26. Moller JT, Cluitmans P, Rasmussen LS, et al. Long-term postoperative cognitive dysfunction in the elderly ISPOCD1 study. ISPOCD investigators. International Study of Post-Operative Cognitive Dysfunction. Lancet 1998;351(9106):857-61.

27. Williams-Russo P, Sharrock NE, Mattis S, Szatrowski TP, Charlson ME. Cognitive effects after epidural vs general anesthesia in older adults. A randomized trial. Jama 1995;274(1):44-50.

28. Marcantonio ER, Flacker JM, Wright RJ, Resnick NM. Reducing delirium after hip fracture: a randomized trial. J Am Geriatr Soc 2001;49(5):516-22.

29. Kalisvaart KJ, de Jonghe JF, Bogaards MJ, et al. Haloperidol prophylaxis for elderly hip-surgery patients at risk for delirium: a randomized placebo-controlled study. J Am Geriatr Soc 2005;53(10):1658-66.

30. Kehlet H, Wilmore DW. Evidence-based surgical care and the evolution of fast-track surgery. Ann Surg 2008;248(2):189-98.

31. Larsen K, Sorensen OG, Hansen TB, Thomsen PB, Soballe K. Accelerated perioperative care and rehabilitation intervention for hip and knee replacement is effective: a randomized clinical trial involving 87 patients with 3 months of follow-up. Acta Orthop 2008;79(2):149-59.

32. Morrison RS, Flanagan S, Fischberg D, Cintron A, Siu AL. A novel interdisciplinary analgesic program reduces pain and improves function in older adults after orthopedic surgery. J Am Geriatr Soc 2009;57(1):1-10. 